\title{
Quantum Noise in Heterodyne Detection
}

\author{
MICHAEL J. WENGLER, STUDENT MEMBER, IEEE, AND DAVID P. WOODY, MEMBER, IEEE
}

\begin{abstract}
A fully quantum mechanical theory of diode mixers which includes quantization of the external circuit is presented. We find that Tucker's theory for SIS mixer conversion efficiencies is correct, but that his expression for the measurement noise must be augmented by an amount corresponding to half a photon at every frequency to which the mixer responds. Noise in high quality SIS mixers is shown to be accurately described by the conceptually simpler photodiode mixer noise theory. The radiation coupling efficiency term, $\eta$, which appears in photodiode theory turns out to be the coupling efficiency between the signal source admittance and the admittance which the SIS presents to the LO. Our theory reduces to Caves' quantum linear amplifier formalism, and therefore predicts measurement noises bounded by the quantum lower limit of $h \nu / k_{\mathbf{B}}$. Predictions of performance versus frequency for SIS's are made. We predict that NbN SIS's will behave as nearly ideal photodiodes for frequencies as high as $\mathbf{3 0 0 0} \mathbf{~ G H z}$.
\end{abstract}

\section{InTRODUCTION}

$\mathrm{S}$ UPERCONDUCTING tunnel junctions (SIS's) provide nearly quantum limited sensitivity for millimeter wavelengths [1]-[4] and have served as practical radioastronomical receivers for many years in this range [2], [5], [6]. First results in the submillimeter suggest that they will be equally sensitive in this range [6], [7]. An elegant quantum mechanical theory for these devices as mixers was developed by John Tucker [8]. The power conversion efficiency predictions of this theory have been verified quantitatively [9]-[11]. Its nonclassical prediction of conversion efficiencies greater than unity have been observed [1], [2], [4]. The development of SIS mixers and mixer theory are reviewed by Tucker and Feldman [12] and by Phillips and Woody [13].

General considerations of the quantum mechanics of heterodyne detection show that for a signal at frequency $\nu$, heterodyne measurement noise has a minimum corresponding to an input noise power $h \nu \mathrm{B}$ where $\mathrm{B}$ is the bandwidth of the measuring circuit [14]-[18]. In contrast to Tucker's device oriented approach, general linear amplifier theories such as Caves' [17] derive the measurement quantum noise limit of $h \nu \mathrm{B}$ by quantizing the electromagnetic fields in the circuit external to the detector without reference to the details of the detection device.

Tucker has developed a theory of mixing in tunnel junctions based upon the microscopic quantum physics of the

Manuscript received September 10, 1986; revised December 8, 1986. This work was supported by the National Science Foundation under Grants AST -8214693 and 8311849 .

M. J. Wengler is with the Department of Applied Physics, California Institute of Technology, Pasadena, CA 91125.

D. P. Woody is with the Owens Valley Radio Observatory, California Institute of Technology, Pasadena, CA 91125.

IEEE Log Number 8613838 . junction, but he does not quantize the external circuit. As a result, his noise predictions approach zero [12], and his theory is thus shown to be incomplete. A complete quantum mechanical description of a mixer must include quantum descriptions of both the device and external circuit to which the device is connected. We fill the theoretical gap between Tucker's and Caves' theories by making a quantum mixing theory in which both a quantized external circuit and the detailed physics of the detector are included. Zorin [19] presents physical results for SIS mixers which agree with those we predict, but he does not present an interpretation of those results in terms of signal and noise. Devyatov et al. [20] present a complete quantum noise theory for the SIS mixer and other devices with results which are consistent with ours. Feldman presents a number of details of SIS mixer noise calculations which we leave out; he also discusses noise in Schottky diode mixers [21].

Our theory looks very much like Tucker's, except voltage and current are represented by operators over states of the device and states of the external circuit. As a result, a simple rescaling of some equations in our theory makes it look like Caves' general amplifier theory. The external circuit is quantized in a similar way to Yurke and Denker's quantum network theory [22]. A mixer output voltage operator is calculated. The output signal corresponds to an expectation value of that operator, $\left\langle\hat{v}_{0}\right\rangle$. For low output frequencies, noise in that measurement is directly calculable as the expected mean square deviation of that measurement from its average, $\left\langle\left(\hat{v}_{0}-\left\langle\hat{v}_{0}\right\rangle\right)^{2}\right\rangle$.

Quantization of the external circuit does not result in different mixer conversion efficiencies from those Tucker predicts, his mixer $Y$ and $Z$ matrices are valid in our fully quantum theory. His noise results also appear in our theory, but they are not complete. We find an additional term which adds noise as though the mixer had down-converted half a photon of power from every sideband to which it responds. This is not a surprising result, it has been recognized as an ad hoc quantum correction to Tucker's theory by others who have discussed mixer noise [12], [21], [23], [24].

What has not been recognized before is that this additional noise term brings SIS mixer theory for ideal SIS's into quantitative agreement with the conceptually simpler theory for photodiode mixers [25], [26]. Photodiode mixer theory gives a quantum noise limit $h \nu \mathrm{B}$, which is entirely attributable to the statistics of the photon absorption process. The agreement of these two theories suggests that photon absorption is the only noise source in an ideal SIS 
mixer as well. Algebraically, this noise appears as a conspiracy of external circuit photon noise and device electron noise. When the device is optimally coupled to the photon field, photon absorption noise is algebraically accounted for by photon operators. When the device is less well-coupled, electron operators for states in the device take up the slack. We appear to be calculating the detailed workings of the Callen and Welton fluctuation-dissipation theorem [27], the quantum limit is enforced by whatever dissipative elements are coupled to the device.

We are mainly interested in SIS mixers for millimeter and submillimeter wavelengths. Once we have presented our theory, we combine it with Tucker's theory to discuss SIS mixer performance. This theory predicts quantum limited performance for good quality SIS mixers up to a limiting frequency which depends on the turn-on voltage of the SIS diode. That upper frequency limit is about 1400 $\mathrm{GHz}$ for lead alloy SIS's, but it may reach $3000 \mathrm{GHz}$ for niobium-nitride junctions which are now being developed for mixers [28]. Our noise results differ from those of Danchi and Sutton [24] because their calculations are done without optimizing the signal source admittance presented to the SIS.

\section{The Quantized External Crrcuit}

We stay in the Heisenberg picture: quantum mechanical states are constant in time, time-evolution of an observable is completely determined by time-evolution of the corresponding operator. This results in equations for voltage and current operators which are quite similar to their classical counterparts. Operators will often be given in terms of their frequency components. The $\omega$ frequency component $\hat{W}_{\omega}(t)$ of an operator $\hat{W}(t)$ will be represented by a time independent "phasor" operator $W_{\omega}$ and its Hermitian conjugate $W_{\omega}^{\dagger}$. These operators for the same observable quantity are related through

$$
\hat{W}_{\omega}(t)=\frac{1}{2}\left(W_{\omega} e^{i \omega t}+W_{\omega}^{\dagger} e^{-i \omega t}\right) .
$$

A "hat" above any operator means that we want the time varying version of that operator, we will sometimes write $\hat{W}(t)$ as $\hat{W}$.

Instead of being considered as a continuous variable, angular frequency $\omega$ is broken into discrete intervals of width $\mathbf{b}$ and summed over. The time varying operators are then given as a sum of the individual frequency components

$$
\hat{W}(t)=\sum_{n=0}^{\infty} \hat{W}_{n b}(t) .
$$

The results we derive are equivalent to those derived by letting $\mathbf{b}$ go to zero, and then integrating over continuous operators to reestablish a nonzero angular bandwidth $\mathbf{b}$, so we skip these algebraic steps. Bandwidth is usually not quoted in angular frequency, so we use

$$
\mathrm{B}=\mathrm{b} / 2 \pi
$$

for bandwidth. For nonzero B, all operators are periodic

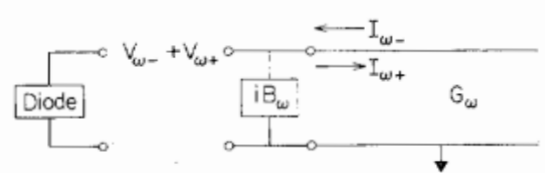

(a)
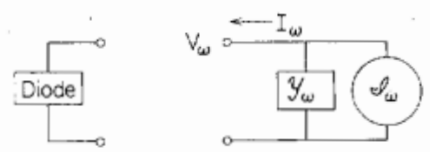

(b)

Fig. 1. (a) The external circuit of the mixer is represented by a semi-infinite transmission line. Reactance from the external circuit appears as a lumped component at the line terminals. The direction of positive current flow for the - and + going waves are shown. (b) An equivalent circuit which is usually used in mixer theories. A linear admittance shunted by a perfect current source models the circuit at each frequency.

in time with period $T=1 / \mathrm{B}$. Measurements separated by a time interval $T$ or longer can be looked at as independent experiments. The average value of these measurements is the expectation value $\langle\hat{W}\rangle$ and the mean square noise is $\left\langle\hat{W}^{2}\right\rangle-\langle\hat{W}\rangle^{2}$.

The external circuit carries photons toward the device, and the device in interacting with these photons generates photons which travel away from the device. The circuit with which we model this process is shown in Fig. 1(a). At every frequency $\omega$ the circuit consists of a reactive lumped component with admittance $i B_{\omega}$ and a transmission line of admittance $G_{\omega}$. Yurke and Denker [22] discuss the quantization of circuits such as those of Fig. 1. They note that the equations of motion for the current and voltage operators are Kirchhoff's circuit laws, the operators evolve in this circuit just as classical voltage and current would.

The voltage and current operators are found following Louisell's transmission line quantization [15]. Quantization is accomplished by considering a length $\mathbf{L}$ of the line with periodic boundary conditions. Allowed frequencies are

$$
\omega=n 2 \pi \mathrm{B} \text { for } n=0,1,2 \cdots
$$

with

$$
\mathrm{B}=c / \mathbf{L}
$$

where $c$ is the speed of light on this transmission line. We choose $\mathrm{L}$ so that this $\mathrm{B}$ is the same as the bandwidth in (2.3). We quantize the incoming $(-)$ and outgoing $(+)$ traveling modes. The Heisenberg photon creation operators for these modes are $\hat{a}_{\omega \alpha}^{\dagger}(t)$ where $\alpha$ takes on values + or - . Letting $a_{\omega \alpha}^{\dagger}$ represent the time independent or Schrödinger picture operator

$$
\hat{a}_{\omega \alpha}^{\dagger}(t)=a_{\omega \alpha}^{\dagger} e^{i \omega t}
$$

shows the explicit time dependence of the Heisenberg operators.

In terms of phasor operators (2.1), voltage and current operators are

$$
\begin{aligned}
V_{\omega \alpha} & =Q_{\omega} a_{\omega \alpha}^{\dagger} \\
I_{\omega \alpha} & =G_{\omega} Q_{\omega} a_{\omega \alpha}^{\dagger}
\end{aligned}
$$


where the "quantum of voltage",

$$
Q_{\omega}=\sqrt{\frac{2 \hbar \omega \mathrm{B}}{G_{\omega}}}
$$

is calculated from (2.5) and Louisell's equation (4.31) [15]. The current and voltage operators at the terminals including the effect of a lumped reactance $i B_{\omega}$ are

$$
\begin{aligned}
& V_{\omega}=V_{\omega-}+V_{\omega+} \\
& I_{\omega}=I_{\omega-}-I_{\omega+}-i B_{\omega} V_{\omega} .
\end{aligned}
$$

Fig. 1(b) shows an equivalent circuit to Fig. 1(a) which is usually used in mixer theory. The two circuits represent the same physical situation if the following relationships between them exist:

$$
\begin{aligned}
\left\langle I_{\omega-}\right\rangle & =\mathscr{I}_{\omega} / 2 \\
\mathcal{Y}_{\omega} & =G_{\omega}+i B_{\omega} .
\end{aligned}
$$

Voltage and current phasor operators are within a constant of the photon operators, just as electric and magnetic field operators are for free-space modes. Their commutation relations are all within a constant of the voltage operator commutators

$$
\begin{aligned}
& {\left[V_{\omega \alpha}, V_{\omega^{\prime} \alpha^{\prime}}\right]=0} \\
& {\left[V_{\omega \alpha}^{\dagger}, V_{\omega^{\prime} \alpha^{\prime}}\right]=\frac{2 \hbar \omega \mathrm{B}}{G_{\omega}} \delta_{\omega, \omega^{\prime}} \delta_{\alpha, \alpha^{\prime}} .}
\end{aligned}
$$

We find the power flux in each mode in terms of the voltage operators. The operator

$$
\begin{aligned}
\hat{V}_{\omega \alpha}^{2}(t)= & \frac{1}{4}\left[V_{\omega \alpha}^{2} e^{i 2 \omega t}+V_{\omega \alpha}^{\dagger 2} e^{-i 2 \omega t}\right. \\
& \left.+V_{\omega \alpha} V_{\omega \alpha}^{\dagger}+V_{\omega \alpha}^{\dagger} V_{\omega \alpha}\right]
\end{aligned}
$$

could be used to define an instantaneous power flow operator. This operator has a time averaged expectation value

$$
\left\langle\hat{V}_{\omega \alpha}^{2}(t)\right\rangle_{t}=\frac{1}{4}\left\langle\left[V_{\omega \alpha}^{\dagger}, V_{\omega \alpha}\right]_{+}\right\rangle
$$

where the subscript $t$ indicates that an average over time $1 / 2 \pi \omega$ is taken to remove terms at frequency $2 \omega$. Anticommutator notation has been introduced for the last two terms of (2.12). The average power flux in each mode is

$$
\begin{aligned}
P_{\omega \alpha} & =G_{\omega}\left\langle\hat{V}_{\omega \alpha}^{2}(t)\right\rangle_{t} \\
& =\frac{G_{\omega}}{4}\left\langle\left[V_{\omega \alpha}^{\dagger}, V_{\omega \alpha}\right]_{+}\right\rangle \\
& =\hbar \omega \mathrm{B}\left(\left\langle\mathrm{n}_{\omega \alpha}\right\rangle+\frac{1}{2}\right)
\end{aligned}
$$

where

$$
n_{\omega \alpha}=a_{\omega \alpha}^{\dagger} a_{\omega \alpha}
$$

is the photon number operator for the mode. The anticommutator was simplified using the voltage commutation re- lation (2.11) and the definition of the voltage phasor (2.7). The quantum of radiation in traveling wave modes is $\hbar \omega \mathrm{B}$ [17].

\section{The Quantized Device}

A heterodyne mixer is made by connecting the terminals of a device to the external circuit of Fig. 1 which supplies signal, LO and dc bias and to which the output signal of the mixer, traditionally called the intermediate frequency (IF), is delivered. Each half of this circuit has a current through it which depends on the voltage waveform across it. Classical mixer theory consists of a consistent solution for both halves of the circuit of

$$
I_{D}^{\mathrm{cl}}\left[V^{\mathrm{cl}}(t)\right]=I_{E}^{\mathrm{cl}}\left[V^{\mathrm{cl}}(t)\right]
$$

where $I_{D}^{\mathrm{cl}}$ and $I_{E}^{\mathrm{cl}}$ give the current through the device and the external circuit respectively as functionals of the voltage waveform. The square brackets in (3.1) are used to indicate that the current at any time may depend on the voltage waveform at the time and earlier. A quantum theory of the external circuit has been described in Section II. The quantum generalization of $I_{D}^{\mathrm{cl}}$ is device dependent, a theory based on the physics of each device to be evaluated as a mixer must be made. We discuss the features this device theory must have for use in this mixer theory.

From (3.1), we see that the device and the external circuit can be treated symmetrically in a mixer theory. A quantized device has a many body electronic state $|D\rangle$, just as the electromagnetic field of the external circuit has a photonic state $|E\rangle$. Let $\hat{V}_{E}$ and $\hat{I}_{E}$ be the voltage and current operators over photon states defined in Section II. A quantum device theory would include, at least implicitly, definitions of operators $\hat{V}_{D}$ and $\hat{I}_{D}$ constructed from the electronic creation and destruction operators. When the device and external circuit are connected at their terminals, $\hat{V}_{E}$ and $\hat{V}_{D}$ are distinct operators over disjoint sets of states, but for the same observable quantity. In fact, this applies to any observable function of these operators. For example, powers of these waveforms are in principle observable, so

$$
\begin{aligned}
\left\langle\hat{V}_{D}^{n}(t)\right\rangle_{D} & =\left\langle\hat{V}_{E}^{n}(t)\right\rangle_{E} \\
\left\langle\hat{I}_{D}^{n}(t)\right\rangle_{D} & =\left\langle\hat{I}_{E}^{n}(t)\right\rangle_{E}
\end{aligned}
$$

for all integer $n$. The subscript $E$ or $D$ on the expectation value are meant to indicate that it is only necessary to evaluate this operator over the external photonic or device electronic states, respectively. The relationship of $|D\rangle$ and $|E\rangle$, which are by no means independent, is implicitly contained in (3.2). These relationships show that it is not necessary in an operator expression to indicate whether a voltage or current operator is over state $|D\rangle$ or $|E\rangle$. We will therefore drop the subscripts $E$ and $D$ from the operators in all further expressions.

To motivate our conditions on the quantum generalization of (3.1), we discuss the causal chain of events in the mixer circuit. At some early time, radiation $|E-\rangle$ is 
moving towards the device which is in some state $|D\rangle$. The radiation is absorbed by the device changing its state to $\left|D^{*}\right\rangle$. The device emits radiation $|E+\rangle$ in returning to its state $|D\rangle$. All mixer equations are presented in the frequency domain, where a time invariant response $|E+\rangle$ is produced by a time invariant stimulus $|E-\rangle$, so the causal chain is not obvious from the algebra.

To generalize (3.1), we consider currents in the device to be driven by voltages from the external circuit, so all voltage operators below are those found in Section II. For a fully quantum theory of (3.1), we require a device theory which allows the calculation of the state $|D\rangle$ associated with every possible state $|E\rangle$ of the external circuit. A unitary operator can be defined so that

$$
|D\rangle=U[\hat{V}(t)]|D 0\rangle
$$

where $|D 0\rangle$ is the state of the device with its leads shorted to each other. The quantum analog of (3.1) is then

$$
\hat{I}_{D 0}(t)=\hat{I}_{D 0}[\hat{V}(t)]
$$

where this operator acts on the equilibrium state $|D 0\rangle$, and is related to the current operator on the left-hand side of (3.2) by

$$
\hat{I}_{D 0}=U^{\dagger} \hat{I} U
$$

When this operator is used instead of $\hat{I}$, the short circuit device state $|D 0\rangle$. should be used instead of $|D\rangle$. In this way a current operator which includes explicit response to voltage is constructed.

Tucker's theory for tunnel junctions [8] very nearly fits the outline above. His current operator corresponding to (3.4) appears in his eq. (2.10). The external circuit in Tucker's mixer theory is not quantized, he therefore uses a classical voltage in place of the voltage operator in (3.4). As a result, his current operator operates only on the device state $|D 0\rangle$ and does not operate on the photon states in $|E\rangle$. Fortunately, quantization of the voltage for a linear mixer theory changes nothing in the device portion of Tucker's device theory, as we will now show.

A tunnel junction consists of a LHS and a RHS electrode, separated by a thin potential barrier. The electronic states in each electrode are assumed to be only mildly perturbed by coupling to the electronic states in the other electrode. This coupling is described by a tunneling Hamiltonian $H_{T}$. The distributions of electrons in the left and right electrodes are assumed to be thermal, so that electronic chemical potentials $\mu_{L}$ and $\mu_{R}$ are defined. The presence of a voltage waveform causes a difference in the chemical potential across the barrier,

$$
\mu_{L}=e V_{\mathrm{cl}}(t)+\mu_{R} .
$$

The unitary matrix $U$ described in (3.3) is not presented explicitly by Tucker, but it is discussed for dc voltages by Rogovin and Scalapino [29]. It is not solved for exactly, it is approximated to second order in $H_{T}$ using perturbation theory. Its voltage dependence, however, is treated exactly through the voltage phasor term

$$
\begin{aligned}
W(t) & =W\left[V_{\mathrm{cl}}(t)\right] \\
& =\exp \left\{-i \frac{e}{\hbar} \int^{t} d t^{\prime} V_{\mathrm{cl}}\left(t^{\prime}\right)\right\} .
\end{aligned}
$$

A heterodyne mixer has large voltages on it due to the $\mathrm{LO}$ and dc bias, which we call $V_{\mathrm{LO}}(t)$. Any other voltages such as those due to signal or noise are assumed to be small enough to deal with only to first order. We define an operator, $\hat{v}$, for these small voltages so that

$$
\hat{V}(t)=V_{\mathrm{LO}}(t)+\hat{v}(t) \text {. }
$$

$V_{\mathrm{LO}}(t)$ is not an operator, it can be interpreted as the portion of $\langle\hat{V}(t)\rangle$ which originates in the LO source. Any fluctuations which originate in the LO source are included in $\hat{v}(t)$.

Tucker's junction is now attached to the quantized external circuit of Section II by putting $\hat{V}(t)$ in place of $V_{\mathrm{cl}}(t)$ in (3.6). The new voltage phasor (3.7) can be factored

$$
W(t)=W\left[V_{\mathrm{LO}}(t)\right] W[\hat{v}(t)]=W_{\mathrm{LO}}(t) W_{v}(t) .
$$

The small voltage operator is a sum of its frequency components as described in (2.1) through (2.3). From (2.11), it is seen that different frequency components of $\hat{v}$ commute, so $W_{v}$ can be factored,

$$
W_{v}=\prod_{\omega} W\left[\hat{v}_{\omega}(t)\right]=\prod_{\omega} W_{v \omega}(t) .
$$

Finally, we find $W_{v \omega}$ to linear order in voltage phasor operators

$$
W_{v \omega}=1-\frac{e}{h \omega}\left(v_{\omega} e^{i \omega t}-v_{\omega}^{\dagger} e^{-i \omega t}\right) .
$$

An important feature of (3.11) is that it has exactly the same form when a small classical voltage is used instead of a voltage operator. The rest of Tucker's development of mixer theory is thus formally applicable to a mixer theory with quantized external circuit by simply interpreting all instances of small voltages in his equations as operators on external photon states. In particular, his expressions for the elements of the mixer $Y$ matrix are unaffected by this quantization. Algebraically, the nonzero commutation relation (2.11) is the only thing that distinguishes small voltage phasor operators from classical small voltage phasors. As a result, terms like $v_{\omega} v_{\omega}^{\dagger}$ and $v_{\omega}^{\dagger} v_{\omega}$ have different meaning in a quantum theory, although they are the same in a classical theory. But for a linear theory, these product terms never appear, so linear classical expressions are algebraically equivalent to linear quantum expressions.

\section{Mixer Output Operator}

In a mixer circuit, the voltage across the device is given by (3.8). The LO is from a coherent radiation source with fundamental angular frequency $\omega_{\mathrm{LO}}$, so $V_{\mathrm{LO}}(t)$ has components at frequencies $m \omega_{\mathrm{LO}}$ for positive integers $m$. We 
also include any dc bias voltage in $V_{\mathrm{LO}}$. Input voltages at the sideband frequencies

$$
\omega_{m}=m \omega_{\mathrm{LO}}+\omega_{0} \quad \text { for all integers } m
$$

are mixed together to produce the mixer output at frequency $\omega_{0}$. We require that $\omega_{0}>0$ and that $\omega_{0} \neq\left|\omega_{m}\right|$ for any integer $m$.

Voltage and current operators have been previously defined for positive frequencies only, so

$$
v_{m}= \begin{cases}V_{\omega_{m}}, & \text { for } \omega_{m} \geq 0 ; \\ V_{-\omega_{m}}^{\dagger} & \text { otherwise }\end{cases}
$$

are the sideband components of $\hat{v}$ in (3.8) where $V_{\omega}$ are the external circuit operators defined in (2.7). External circuit sideband current operators $i_{m}$ are similarly defined, as are device current operators $I_{D O m}$ for the components of (3.4). The external source admittances are

$$
\mathcal{Y}_{m}= \begin{cases}\mathcal{Y}_{\omega_{m}}, & \text { for } \omega_{m} \geq 0 \\ \mathcal{Y}_{-_{\omega_{m}}}^{*} & \text { otherwise }\end{cases}
$$

Determining the mixer response is a matter of calculating the current operator in (3.4) as a function of the voltage operator in (3.8). Treating $\hat{v}$ as a perturbation on the device in the $U\left[V_{\mathrm{LO}}\right]|D 0\rangle$ state, the sideband current phasor operators are found as a first order perturbation

$$
\begin{aligned}
i_{m} & =I_{D 0 m}\left[V_{\mathrm{LO}}(t)\right]+\sum_{m^{\prime}} Y_{m m^{\prime}}\left[V_{\mathrm{LO}}(t)\right] v_{m^{\prime}} \\
& =I_{\mathrm{LO} m}+\sum_{m^{\prime}} Y_{m m^{\prime}} v_{m^{\prime}} .
\end{aligned}
$$

$I_{\mathrm{LO} m}$ and $Y_{m m^{\prime}}$ are operators over $|D 0\rangle$ only, they do not operate on $|E\rangle$. Their fundamental dependence on $V_{\mathrm{LO}}$ is shown explicitly in (4.4), but will not be shown through the rest of this paper. We need consider only the expectation value of $Y_{m m^{\prime}}$, since the fluctuating component of $Y_{m m}$ 'corresponds to gain fluctuations which are explicitly ignored in a linear theory [17]. Without changing the symbol, we interpret $Y_{m m^{\prime}}$ as an expectation value from here on.

Relating Tucker's junction device theory with our general notation, we have

$$
I_{\mathrm{LO} m}=4 \pi \mathrm{B} I_{T}\left(-\omega_{m}\right) \text { for } T=1 / \mathrm{B}
$$

where $I_{T}$ is the current operator in Tucker's equation (7.9). This operator represents the sideband current flowing due to the LO voltage waveform. Its expectation value must be zero, since the expected current waveform must, like $V_{\mathrm{LO}}$, have components only at $\omega_{\mathrm{LO}}$ and its harmonics. Its fluctuations, however, have components at all frequencies.

The output voltage wave operator is found by comparing current operators in (4.4) and (2.9). In matrix and vector notation, (4.4) becomes

$$
\vec{i}=\vec{I}_{\mathrm{LO}}+Y \vec{v} .
$$

The sideband current and voltage operators can be broken down into their + and - going components using (2.9) and (2.10). Rearranging terms

$$
(Y+Y) \vec{v}_{+}=-\vec{I}_{\mathrm{LO}}+\left(\mathcal{Y}^{*}-Y\right) \vec{v}_{-}
$$

where $\mathcal{Y}_{m m^{\prime}}$ is the diagonal matrix of $\mathcal{Y}_{m}$ values. In traditional mixer theory, the matrix $(\mathcal{Y}+Y)$ has an inverse matrix called $Z$, for example see Tucker's equation (6.8). We multiply both sides of (4.7) by this matrix

$$
\vec{v}_{+}=\Gamma \vec{v}_{-}-Z \vec{I}_{\mathrm{LO}}
$$

where

$$
\begin{aligned}
\Gamma & =Z\left(\mathcal{Y}^{*}-Y\right) \\
& =2 Z G-\mathbf{I} .
\end{aligned}
$$

I is the identity matrix and the $G$ matrix is the real part of $Y$. Abandoning matrix notation, we have the output voltage operator

$$
\begin{aligned}
v_{0+} & =\sum_{m}\left(\Gamma_{0 m} v_{m-}-Z_{0 m} I_{\mathrm{LO} m}\right) \\
& =-v_{0-}+\sum_{m} Z_{0 m}\left(2 G_{m} v_{m-}-I_{\mathrm{LO} m}\right) .
\end{aligned}
$$

The output photon state $|E 0+\rangle$ is implicitly determined by the states $|E-\rangle$ and $|D 0\rangle$ by this equation, as one would expect from the causal chain described in Section III. Equation (4.8) defines the output voltage wave at all frequencies except harmonics of the LO. Of course, the matrices and operators with subscripts $m$ must be reevaluated for each choice of $\omega_{0}$ since $m$ is a shorthand for $\omega_{0}+m \omega_{\mathrm{LO}}$.

The output power (2.14) of the mixer is

$$
P_{0+}=\frac{G_{0}}{4}\left\langle\left[v_{0+}, v_{0+}^{\dagger}\right]_{+}\right\rangle_{E+} .
$$

Using (4.10), the expectation value of the anticommutator can be evaluated over states $|D 0\rangle$ and $|E-\rangle$

$$
\begin{aligned}
\left\langle\left[v_{0+}, v_{0+}^{\dagger}\right]_{+}\right\rangle_{E+}= & \sum_{m m^{\prime}} Z_{0 m} Z_{0 m^{\prime}}^{*}\left\langle\left[I_{\mathrm{LO} m}, I_{\mathrm{LO} m^{\prime}}^{\dagger}\right]_{+}\right\rangle_{D 0} \\
& +\sum_{m m^{\prime}} \Gamma_{0 m} \Gamma_{0 m^{\prime}}^{*}\left\langle\left[v_{m-}, v_{m^{\prime}-}^{\dagger}\right]_{+}\right\rangle_{E-} .
\end{aligned}
$$

Cross terms are not written,

$$
\left\langle\left[v_{m-}, I_{\mathrm{LO} m^{\prime}}^{\dagger}\right]_{+}\right\rangle_{E-, D 0}=2\left\langle v_{m-}\right\rangle_{E-}\left\langle I_{\mathrm{LO}^{\prime}}^{\dagger}\right\rangle_{D 0}=0
$$

because $\left\langle I_{\mathrm{LO} m^{\prime}}^{\dagger}\right\rangle_{D 0}$ is zero.

The first sum term in (4.12) accounts for output power which Tucker associates with noise in his mixer theory. To indicate that it accounts only for noise associated with the device operators, we label the current correlation matrix that Tucker calls $H$ in his equation (7.14) $H_{D}$. Using (4.5) to calculate this in terms of our operators

$$
H_{D m m^{\prime}}=\frac{1}{4 \mathrm{~B}}\left\langle\left[I_{\mathrm{LO} m}, I_{\mathrm{LO} m^{\prime}}^{\dagger}\right]_{+}\right\rangle_{D 0^{\circ}}
$$


The second sum in (4.12) accounts for output power due to interaction with the incoming radiation. A current correlation matrix for the incoming radiation is

$$
\begin{aligned}
H_{E m m^{\prime}}= & \frac{G_{m} G_{m^{\prime}}\left\langle\left[v_{m-}, v_{m^{\prime}-}^{\dagger}\right]_{+}\right\rangle_{E-}}{\mathrm{B}} \\
= & \begin{cases}\frac{2 G_{m} G_{m^{\prime}}}{\mathrm{B}}\left\langle v_{m-}\right\rangle_{E-}\left\langle v_{m^{\prime}-}^{\dagger}\right\rangle_{E-} & \text { for } m \neq m^{\prime} \\
4 \hbar \omega_{m} G_{m}\left\langle n_{m-}+\frac{1}{2}\right\rangle_{E-} & \text { for } m=m^{\prime} .\end{cases}
\end{aligned}
$$

The off-diagonal element $H_{E m m^{\prime}}$ is nonzero only if the photon states $|E m-\rangle$ and $\left|E m^{\prime}-\right\rangle$ are correlated. This occurs when, for instance, the radiation source is a parametric amplifier pumped with radiation which is phaselocked to the mixer LO [17]. For most physical situations, $H_{E}$ is diagonal and the mixer output power is

$$
\begin{aligned}
P_{0+}= & G_{0} \mathrm{~B} \sum_{m m^{\prime}} Z_{0 m} Z_{0 m^{\prime}}^{*} H_{D m m^{\prime}} \\
& +\mathrm{B} \sum_{m} \mathcal{G}_{0 m}\left\langle n_{m-}+\frac{1}{2}\right\rangle_{E-} \hbar \omega_{m} .
\end{aligned}
$$

$\mathrm{S}_{0 m}$ is the mixer conversion efficiency,

$$
\mathcal{G}_{0 m}=\frac{G_{0}}{G_{m}}\left|\Gamma_{0 m}\right|^{2}= \begin{cases}4 G_{0} G_{m}\left|Z_{0 m}\right|^{2} & \text { for } m \neq 0 \\ \left|1-2 G_{0} Z_{00}\right|^{2} & \text { for } m=0 .\end{cases}
$$

We have made no attempt to describe the apparatus which measures $P_{0+}$. A photon counting power meter measures $n_{\omega_{0}}$ and detects $\hbar \omega_{0} \mathrm{~B} / 2$ less than (2.14) predicts, while a classical power meter following a high gain linear amplifier would detect $\hbar \omega_{0} \mathrm{~B} / 2$ more than our power expression. For the important special case that $\omega_{0}$ $<<\omega_{L O}$, these differences are negligible, and (2.14) describes the results of any type of power measurement accurately. For this case, our theory is a complete theory for heterodyne measurement. For larger output frequencies, the details of the output measuring system must be considered.

If $\omega_{0}$ is small, we can also set $P_{0-}$ to zero. Then the $v_{0-}$ term in (4.10) does not contribute to the mixer output power, so (4.11) becomes

$$
P_{0+}=G_{0} \mathrm{~B} \sum_{m m^{\prime}} Z_{0 m} Z_{0 m^{\prime}}^{*}\left(H_{D m m^{\prime}}+H_{E m m^{\prime}}\right) \text { for } P_{0-}=0 \text {. }
$$

Most of Tucker's expressions are derived under these assumptions. Tucker's mixer noise expressions involve sums like (4.18) over an $H$ matrix which is equivalent to $H_{D}$. As we have defined $H_{E}$ in (4.15), it accounts for mixer response to signal power, but as we show below, it also includes an additional noise term which completes tunnel junction mixer noise theory.

Equation (4.10) is close to the formalism used by Caves
[17] for consideration of lower limits on noise in amplifiers. Caves' equation (3.5) relates the output signal photon operator to input signal photon operators and a noise operator $\mathfrak{F}_{0}$ through

$$
a_{0+}=\sum_{m \in S}\left(M_{0 m} a_{\left|\omega_{m}\right|-}+L_{0 m} a_{\left|\omega_{m}\right|-}^{*}\right)+\mathscr{F}_{0}
$$

where $S$ is the set of sidebands which carry signal. This is equivalent to (4.10) if

$$
\begin{aligned}
& M_{0 m}= \begin{cases}\sqrt{\frac{\left|\omega_{m}\right| G_{0}}{\omega_{0} G_{m}} \Gamma_{0 m}^{*}} & \text { for } \omega_{m}>0 \\
0 & \text { otherwise }\end{cases} \\
& L_{0 m}= \begin{cases}\sqrt{\frac{\left|\omega_{m}\right| G_{0}}{\omega_{0} G_{m}} \Gamma_{0 m}^{*}} & \text { for } \omega_{m}<0 \\
0 & \text { otherwise }\end{cases} \\
& \mathfrak{F}_{0}= \sum_{m} \sqrt{\frac{\left|\omega_{m}\right| G_{0}}{\omega_{0} G_{m}} Z_{0 m}^{*} I_{L O m}}+\sum_{m \notin \mathrm{S}}\left(M_{0 m} a_{\left|\omega_{m}\right|-}\right. \\
&\left.+L_{0 m} a_{\left|\omega_{m}\right|-}^{\dagger}\right) .
\end{aligned}
$$

The $\Gamma_{0 m}^{*}$ for positive $\omega_{m}$ correspond to Caves' phase-preserving gain terms $M_{0 m}$, while negative $\omega_{m}$ produce phaseconjugated gain $L_{0 m}$. Caves refers to the modes which are summed over to produce $\mathcal{F}_{0}$ as internal modes. As he points out just before his conclusion, sideband modes which do not carry signal must be included with the noiseadding internal modes of the device.

Since our formalism is equivalent to Caves', it is consistent with the quantum limits Caves' has derived for linear amplifiers. Although we are not going to pursue the interesting field of quantum nondemolition measurements in this paper, it is clear that (4.10) can be used to handle correlated photon input states (squeezed states) to heterodyne mixers.

\section{Mixer Gain and Noise}

In a classical theory, voltages can be measured instantaneously and exactly, so classical receiver theories use voltage and signal interchangeably. Quantum mechanically, the measurement of voltage is distinguished from voltage itself, so signal must be identified with the expectation value of the voltage waveform. The simplest use of a mixer is to determine the input voltage phasor in one sideband by measuring the output voltage phasor. For convenience we choose the $m=1$ sideband to carry the signal. The input and output signals are

$$
\begin{aligned}
V_{\mathrm{IN}} & =\left\langle v_{1-}\right\rangle \\
V_{\text {OUT }}=\left\langle v_{0+}\right\rangle & =\Gamma_{01} V_{\mathrm{IN}}
\end{aligned}
$$

where $\Gamma_{01}$ in (4.10) is now identified as the voltage gain of the mixer. It is common to express mixer performance 
in terms of powers. The signal powers are

$$
\begin{gathered}
P_{\mathrm{IN}}=\frac{1}{2} G_{1}\left|V_{\mathrm{IN}}\right|^{2} \\
P_{\text {OUT }}=\frac{1}{2} G_{0}\left|V_{\text {OUT }}\right|^{2}
\end{gathered}
$$

and they are linearly related by the conversion gain of the mixer (4.17)

$$
P_{\text {OUT }}=S_{01} P_{\mathrm{IN}} .
$$

Noise in the voltage measurement is characterized by the expected mean square deviation of a set of voltage measurements from the true expectation value. The voltage phasor operator is not an observable operator, so noise is calculated in the time domain using (2.13)

$$
\begin{aligned}
V_{N}^{2} & =\left\langle\left(\hat{v}_{0+}(t)-\left\langle\hat{v}_{0+}(t)\right\rangle\right)^{2}\right\rangle \\
& =\frac{1}{4}\left(\left\langle\left[v_{0+}, v_{0+}^{*}\right]_{+}\right\rangle-V_{\text {OUT }}^{*} V_{\text {OUT }}\right) \\
P_{N} & =G_{0} V_{N}^{2} .
\end{aligned}
$$

The minimum detectable signal power is defined to be the signal power which results in an output signal to noise ratio (SNR) of unity

$$
P_{\text {det }}=P_{N} / G_{01} \text {. }
$$

The minimum detectable power is generally proportional to the bandwidth $\mathbf{B}$, so the noise figure of merit for a mixer is a detectable noise power per bandwidth

$$
E_{M}=P_{\text {det }} / \text { B. }
$$

Traditionally, this is presented in units of temperature

$$
T_{M}=E_{M} / k_{B}
$$

but it can also be expressed in units of photons

$$
N_{M}=E_{M} / \hbar \omega_{1} \text {. }
$$

We now work out the noise for this measurement. Assume that the signal state $|E 1-\rangle$ is an eigenstate of $v_{1-}^{\dagger}$ with eigenvalue $V_{\mathrm{IN}}$. Since $v_{1-}^{\dagger}$ is proportional to $a_{1-}$, this eigenstate is a Glauber coherent state. This state carries power (2.14)

$$
\begin{aligned}
P_{1-} & =\frac{G_{1}}{4}\left\langle V_{\mathrm{IN}}\left|\left[v_{1-}^{\dagger}, v_{1-}\right]_{+}\right| V_{\mathrm{IN}}\right\rangle \\
& =\frac{G_{1}}{2}\left\langle V_{\mathrm{IN}}\left|v_{1-} v_{1-}^{\dagger}\right| V_{\mathrm{IN}}\right\rangle+\hbar \omega_{1} \mathrm{~B} / 2 \\
& =\frac{G_{1}}{2}\left|V_{\mathrm{IN}}\right|^{2}+\hbar \omega_{1} \mathrm{~B} / 2 \\
& =P_{\mathrm{IN}}+\hbar \omega_{1} \mathrm{~B} / 2
\end{aligned}
$$

where (2.11) was used to expand the anticommutator. Comparison with (2.14) shows that photons counted by the number operator $n_{1}$ - account exactly for signal power in this state, but power flux exceeds signal power by an unavoidable half photon which we attribute to the vac- uum. To calculate minimum mixer noise, assume that all other incoming radiation states are in their vacuum states. Subtracting output signal power (5.3) from the total output power (4.16), we have the output noise power

$$
P_{N}=G_{0} \mathrm{~B} \sum_{m m^{\prime}} Z_{0 m} Z_{0 m^{\prime}}^{*} H_{D m m^{\prime}}+\mathrm{B} \sum_{m} \mathrm{~S}_{0 m} \frac{1}{2} \hbar \omega_{m} .
$$

The first term above is the noise term which Tucker derives for tunnel diode mixers. The second term is due to quantization of the external circuit, and it represents the minimum noise possible for the signal in one sideband only, since $\left\langle n_{m-}\right\rangle \geq 0$. For this mixer, the vacuum half photon at every sideband appears to be frequency converted into noise power at the mixer output.

The nonsignal sidebands may be in thermal equilibrium photon states. The $m$ th sideband photon state in thermal equilibrium at temperature $T_{m}$ has

$$
\begin{aligned}
\left\langle v_{m}\right\rangle_{T_{m}} & =0 \\
\left\langle n_{m-}+\frac{1}{2}\right\rangle_{T_{m}} & =\frac{1}{2} \operatorname{coth}\left(\hbar \omega_{m} / 2 k_{B} T_{m}\right)
\end{aligned}
$$

[16], [27] which has limiting forms

$$
\left\langle n_{m-}+\frac{1}{2}\right\rangle= \begin{cases}\frac{1}{2} & \text { for } T_{m}=0 \\ \frac{k_{B} T_{m}}{\hbar\left|\omega_{m}\right|} & \text { for } k_{B} T_{m} \gg \hbar\left|\omega_{m}\right| .\end{cases}
$$

These limits are the vacuum state and the Rayleigh-Jeans limit, respectively. The noise in (5.10) represents the case that all unused sidebands are terminated at $0 \mathrm{~K}$. We can include the effect of finite temperature termination of nonsignal sidebands by replacing the $1 / 2$ in the second term on the right of (5.10) with the second line in (5.11).

Tucker suggests in his equation (7.8) that the Planck formula

$$
\left\langle n_{m-}\right\rangle_{T_{m}}=\frac{1}{\exp \left(\hbar \omega_{m} / k_{B} T_{m}\right)-1}
$$

be used to describe thermal noise. This is the traditional formula for the available photons, it does not include the unavailable one-half photon in (5.11) that is attributed to the vacuum. This relation between the Planck formula and (5.11) serves to emphasize the identification of the second term on the right of (5.10) as the quantum noise term. Amplifier gain and noise measurements are often made using black bodies at various known temperatures as calibrated power sources. Since one-half photon of (5.11) must always be identified with unavoidable noise, the Planck formula (5.13) should be used to describe thermal "signals," even for high temperatures. Use of the Rayleigh-Jeans approximation (5.12) to describe hot blackbodies results in the underestimation of mixer noise by about one-half photon. 


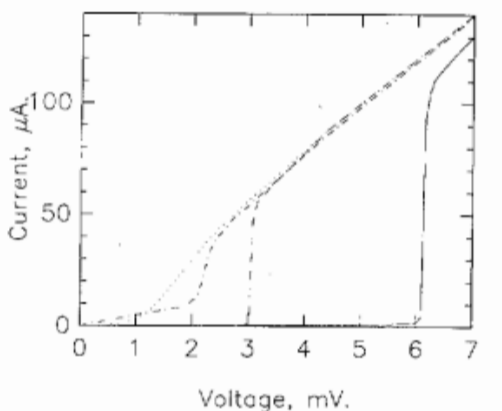

Fig. 2. Current-voltage curves for four different tunnel junctions. The dotted line is for an SIN at $1.6 \mathrm{~K}$. The dashed and dot-dash lines are for lead alloy SIS's at 2.5 and $4.3 \mathrm{~K}$, respectively. The solid line is made by scaling voltage and current from the dashed line to represent a niobium-nitride SIS, which are currently under development for submillimeter receivers.

\section{SIS MiXers}

We have developed the theory above very generally so it can serve as a framework for a quantum mixer theory of any device. The inclusion of the external circuit noise from $H_{E}$ is not device dependent at all, nor is the fact that the $Y$ and $Z$ matrices relate voltage and current operators in the external circuit. However, our formalism has been developed so that it will fit with the formalism developed by Tucker for tunnel diodes, and complete the noise theory of these devices. Having done this, we now discuss the performance of SIS mixers.

A computer program has been written which calculates mixer performance based on Tucker's theory for the conversion efficiency and device noise and the complete noise as given by (5.10) using measured dc current-voltage curves. In Fig. 2 are shown the current-voltage relationships for four different tunnel junctions. The dashed line is from a lead alloy junction cooled to $2.5 \mathrm{~K} \mathrm{[30].} \mathrm{The}$ dot-dash line is for the lead alloy junction which was measured from 115 to $466 \mathrm{GHz}$ by Wengler et al. [7] cooled to $4.3 \mathrm{~K}$. The dotted line is for a superconductor-insulator-normal metal (SIN) junction cooled to $1.6 \mathrm{~K}$ [31]. For the sake of comparison, the measured currents of these three junctions have been scaled so that all three would appear to be $50 \Omega$ resistors above their superconducting transitions. In addition, an artificial curve is drawn for the hoped for niobium nitride SIS, which has a significantly higher $V_{G}$ ("gap voltage" at which current starts flowing) than lead based junctions [28]. Predictions of single sideband mixer performance have been made for these four junctions assuming that $m=1$ and -1 sidebands have the same terminating admittance. The assumptions made are $\omega_{0}$ is small, nonsignal sidebands are at $0 \mathrm{~K}$, and Johnson noise from the SIS's is not included. The noise performance of these receivers is optimized against dc bias voltage, the LO drive voltage $V_{\mathrm{LO}}$, and the signal source admittance $\mathcal{Y}_{1}$.

The results are shown in Fig. 3. The warmer SIS and the SIN do no better than $N_{M}$ of a few, due to their nonzero "dark currents." It is interesting to note that the rather mediocre warm SIS is predicted to outperform a

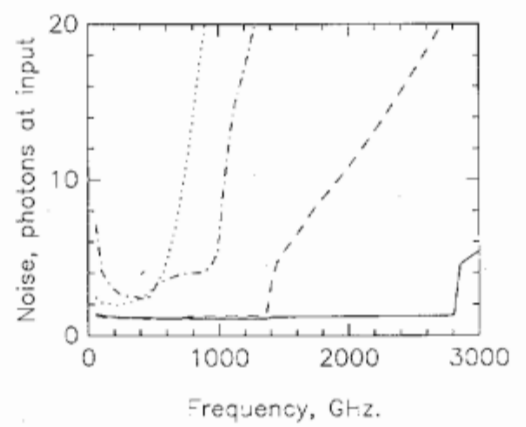

Fig. 3. Mixer noise under optimum conditions versus frequency predicted for the four devices of Fig. 2.

much colder SIN for $400-1000 \mathrm{GHz}$, because of its intrinsically more nonlinear SIS current-voltage relation and its higher $V_{G}$. Each device degrades above some high frequency $f_{U} \sim 2 V_{G} / h$. The colder lead and the fictitious NbN SIS's are close to the quantum limit of one photon up to $f_{U}$. We conclude that SIS's should provide quantum limited detection up to about $1400 \mathrm{GHz}$ for lead alloys, and to as high as $3000 \mathrm{GHz}$ for $\mathrm{NbN}$.

In general the calculation of mixer performance requires knowing all of the terminating admittances and Tucker's full formalism in combination with (5.10) must be used. Fortunately the performance is easy to characterize in the interesting situation where the noise approaches the quantum limit. Quantum limited performance is achieved when and only when the leakage or "dark current" is small and the absorption of one photon produces one electron in the output current [21], that is when the device behaves like an ideal photodiode. This is the case for an ideal SIS tunnel junction in the limit of low LO power. In this case, the expressions that Tucker gives for the $Y$ and $H_{D}$ matrices can be simplified, see for example, [12], [21], [24], and the mixer noise given by (5.10) is simply the inverse of the coupling efficiency as it is for more traditional photodiodes,

$$
N_{M}=1 / \eta
$$

where

$$
\eta=\frac{4 G_{\mathrm{LO}} G_{1}}{\left|\mathcal{Y}_{\mathrm{LO}}+\mathfrak{Y}_{1}\right|^{2}}
$$

and

$$
\begin{aligned}
y_{\mathrm{LO}} & =\frac{I_{\mathrm{LO}}}{V_{\mathrm{LO}}} \\
G_{\mathrm{LO}} & =\operatorname{Re} Y_{\mathrm{LO}} .
\end{aligned}
$$

$V_{\mathrm{LO}}$ and $I_{\mathrm{LO}}$ are the voltage and current phasors at the LO frequency, $I_{\mathrm{LO}}$ can be calculated from Tucker's equation (5.2). $\eta$ is the coupling efficiency between a source admittance $\mathcal{Y}_{1}$ and a load admittance $\mathcal{Y}_{\text {LO }}$.

Note that the optimum source admittance is the admittance which the device presents to the LO and not the admittance it presents at the signal frequency. The load admittance which the SIS mixer presents to the input ra- 
diation at $m=1$ can be calculated from the $Y$ matrix and the terminating admittances $\mathcal{Y}_{m}$. In general, that admittance is not equal to $\mathfrak{Y}_{\mathrm{LO}}$, which is completely independent of all $\mathcal{Y}_{m}$. McGrath has suggested to us that the $m=$ 1 mixer load admittance can be derived from an active circuit with a feedback loop [32]. $\mathcal{Y}_{\text {LO }}$ is apparently the passive admittance in this circuit. It is therefore this admittance to which Callen and Welton's fluctuation-dissipation theorem [27] applies, and this admittance which the external circuit must match to minimize measurement noise.

This equation for mixer temperature is independent of $\mathcal{Y}_{-1}$ [21]. Consider the optimum mixer with $N_{M}=1$ at $\mathcal{Y}_{1}=\mathcal{Y}_{\mathrm{LO}}^{*}$. If the image $(m=-1)$ is also optimally coupled to the mixer, then $\mathcal{Y}_{-1}=\mathcal{Y}_{\mathrm{LO}}$ and all of $N_{M}=$ 1 is algebraically due to the $H_{E}$ term in (5.10). If, however, $\mathcal{Y}_{-1}$ is purely reactive, then half of $N_{M}$ comes from $H_{E}$ and half comes from Tucker's term, $H_{D}$. We are seeing the detailed workings of the fluctuation-dissipation theorem [27]. When the $\mathcal{Y}_{-1}$ is well coupled to the mixer, its dissipation enforces the quantum limit, but decoupling $\mathcal{Y}_{-1}$ from the mixer does not lower the quantum limit, instead dissipation in the device provides the necessary noise.

Equation (6.1) is the same as that obtained for a perfect photodiode. Their properties as heterodyne mixers are discussed in textbooks by Kingston [25] and Marcuse [26]. For photodiode theories, the external radiation source is not allowed to vary over the RF frequency range including the signal, LO, and image. A similar constraint on the external circuit of an SIS mixer corresponds to holding $Y_{\omega}$ in Fig. 2 constant over the range $\left|\omega_{-1}\right|<\omega$ $<\omega_{1}$. In this case, $\eta$ defined in (6.2) is the fraction of incident LO radiation which is absorbed by the SIS diode. This is precisely the definition of $\eta$ in photodiode mixer theories. When (6.1) is derived for a photodiode mixer, it is calculated from the statistics associated with LO photon absorption. The fact that we derive this same amount of noise for an ideal SIS mixer leads us to conclude that, as in a photodiode mixer, noise in an ideal SIS mixer can ultimately be identified with the statistics of the photon absorption process. We conclude that not only is an ideal SIS tunnel junction like a perfect photodiode in its response, producing one electron in its output current for each photon absorbed as Tucker has shown, but that it is like a perfect photodiode in its noise properties as well.

Real SIS mixers are not operated at low LO powers. One effect of a nonzero LO power is that sidebands $|m|>1$ may play a role in mixer performance, but we will assume that these sidebands are shorted out by the circuit surrounding the tunnel junction. An effect of the higher LO power that cannot be neglected is that the SIS response to the LO saturates; not all absorbed photons result in the conduction of electrons across the device. While (6.1) is no longer exact for this case, we show that it is still a good approximation. Computer calculations of (5.10) have been done for a nearly ideal SIS with the realistic values $e V_{\mathrm{LO}}=\hbar \omega_{\mathrm{LO}}=e V_{G}$ and a variety of con-

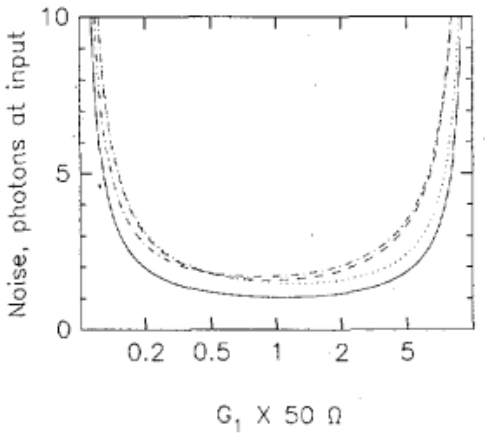

Fig. 4. Mixer noise $N_{M}$ versus real source admittance for an SIS with large LO power. The junction used is the $2.5 \mathrm{~K}$ SIS of Fig. 2. The dashed, dotted and dash-dot lines are for image termination equal to signal termination, open circuit, and short circuit, respectively. The solid line is a plot of (6.1) for comparison. The $x$-axis is the zero reactance slice through a standard Smith chart.

ditions on the image termination $\mathcal{Y}_{-1}$. This value of LO voltage represents more absorbed LO power than we typically find necessary in our actual mixers [5], [7]. These results are shown in Fig. 4, along with a calculation of (6.1) for comparison. The image termination $\mathcal{Y}_{-1}$ slightly effects mixer noise for this high LO power, but simple photodiode theory is still a good approximation to (5.10). Of course, for nonideal junctions, the full expression (5.10) must be used, and (6.1) will not be very accurate.

\section{Conclusions}

We have produced a fully quantum mixer theory which includes all noise effects in a heterodyne measurement. For the standard use of a mixer, we present expressions for mixer gain and noise. These are derived from the expectation value of an output voltage operator and its square. Photodiode mixer theory is shown to be sufficient for describing noise in high quality SIS mixers. The operator formalism presented here can be easily extended to make predictions of SIS response to correlated photon states, which are the subject of much current research.

Our output operator expressions are similar to Caves', so his limits on amplifier performance should apply to any mixer analyzed with our theory. We have shown that SIS mixers can reach this limit when analyzed using Tucker's and our theories together. In fact, we have shown that ideal SIS mixer noise is predicted by the simple expressions of photodiode mixer theory, and is simply related to how well radiation is coupled to the SIS. We have calculated SIS and SIN mixer performance versus frequency and these calculations suggest that quantum limited detection should be possible to as high as $3000 \mathrm{GHz}$ with niobium nitride SIS's which are now being developed.

\section{ACKNOWLEDGMENT}

We are grateful to E. Grossman for many days of discussion which improved our understanding of photon operators. Discussions with D. Watson, E. Brown, B. Schumaker, T. Phillips, and J. Stern have contributed to this 
work. We thank J. Tucker for removing errors from this work before we published them.

\section{REFERENCES}

[1] W. R. McGrath, P. L. Richards, A. D. Smith, H. van Kempen, and R. A. Batchelor, "Large gain, negative resistance, and oscillations in superconducting quasiparticle heterodyne mixers," Appl. Phys. Lett., vol. 39, pp. 655-658, Oct. 15, 1981.

[2] A. A. Stark and R. E. Miller, private communication.

[3] L. R. D'Addario, "An SIS mixer for 90-120 ghz with gain and wide bandwidth," Int. J. IR, Millimeter Waves, vol. 5, pp. 1419-1442, 1985.

[4] D. W. Face, D. E. Prober, W. R. McGrath, and P. L. Richards, "High quality tantalum superconducting tunnel junctions for microwave mixing in the quantum limit," Appl. Phys. 'Lett., vol. 48, pp. 1098-1100, Apr. 21, 1986.

[5] D. P. Woody, R. E. Miller, and M. J. Wengler, " $85-115 \mathrm{GHz}$ receivers for radio astronomy, ' IEEE Trans. Microwave Theory Tech., vol. MTT-33, pp. 90-95, Feb. 1985.

[6] E. C. Sutton, "A superconducting tunnel junction receiver for 230 GHz," IEEE Trans. Microwave Theory Tech., vol. MTT-31, pp. 589592, July 1983.

[7] M. J. Wengler, D. P. Woody, R. E. Miller, and T. G. Phillips, "A low noise receiver for millimeter and submillimeter wavelengths," Int. J. IR, Millimeter Waves, vol. 6, pp. 697-706, Aug. 1985.

[8] J. R. Tucker, "Quantum limited detection in tunnel junction mixers," IEEE J. Quantum Electron., vol. QE-15, pp. 1234-1258, Nov. 1979.

[9] M. J. Wengler and D. P. Woody, in T. G. Phillips and G. J. Dolan, "SIS mixers," Physica, vols. 109 and 110B, pp. 2010-2019, July 1982.

[10] M. J. Feldman and S. Rudner, "Mixing with SIS arrays," in Reviews of Infrared and Millimeter Waves, Vol. 1, K. J. Button, Ed. New York: Plenum, 1983.

[11] M. J. Feldman, S.-K. Pan, A. R. Kerr, and A. Davidson, "'SIS mixer analysis using a scale model," IEEE Trans. Magn., vol. MAG-19, pp. 494-497, May 1983.

[12] J. R. Tucker and M. J. Feldman, "Quantum detection at millimeter wavelengths,"' Rev. Modern Phys., vol. 57, pp. 1055-1113, Oct. 1985.

[13] T. G. Phillips and D. P. Woody, "Millimeter- and submillimeterwave receivers," Ann. Rev. Astron. Astrophys., vol. 20, pp. 285$321,1982$.

[14] H. Heffner, "The fundamental noise limit of linear amplifiers," in Proc. Inst. Radio Eng., vol. 50, p. 1604, July 1962.

[15] W. H. Louisell, Radiation and Noise in Quantum Electronics. New York: McGraw-Hill, 1964, sect. 4.3

[16] F. N. H. Robinson, Noise and Fluctuations in Electronic Devices and Circuits. Oxford, England: Clarendon, 1974.

[17] C. M. Caves, "Quantum limits on noise in linear amplifiers,"'Phys. Rev. $D$, vol. 26 , pp. $1817-1839$, Oct. 15,1982

[18] J. H. Shapiro and S. S. Wagner, "Phase and amplitude uncertainties in heterodyne detection," IEEE J. Quantum Electron., vol. QE-20, pp. 803-813, July 1984 .

[19] A. B. Zorin, "Quantum noise in SIS mixers," IEEE Trans. Magn., vol. MAG-21, pp. 939-942, Mar. 1985.

[20] I. A. Devyatov, L. S. Kuzmin, K. K. Likharev, V. V. Migulin, and A. B. Zorin, "Quantum-statistical theory of microwave detection using superconducting tunnel junctions," J. Appl. Phys., vol. 60, pp. 1808-1828, Sept. 1986.

[21] M. J. Feldman, "Quantum noise in the quantum theory of mixing," IEEE Trans. Magn., vol. MAG-23, 1987.

[22] B. Yurke and J. S. Denker, "Quantum network theory," Phys. Rev. $A$, vol. 29, pp. 1419-1437, Mar. 1984.

[23] H. J. Hartfuß and M. Tutter, "Minimum noise temperature of a practical SIS quantum mixer," Int. J. IR, Millimeter Waves, vol. 5, pp. 717-735, May 1984.
[24] W. C. Danchi and E. C. Sutton, "Frequency dependence of quasiparticle mixers," J. Appl. Phys., vol. 60, pp. 3967-3978, Dec. 1, 1986.

[25] R. H. Kingston, Detection of Optical and Infrared Radiation. New York: Springer-Verlag, 1978, ch. 3.

[26] D. Marcuse, Principles of Quantum Electronics. New York: Academic, 1980.

[27] H. B. Callen and T. A. Welton, "Irreversibility and generalized noise," Phys. Rev., vol. 83, pp. 34-40, July 1951.

[28] H. G. LeDuc, J. A. Stern, S. Thakoor, and S. Khanna, "All refractory $\mathrm{NbN} / \mathrm{MgO} / \mathrm{NbN}$ tunnel junctions," IEEE Trans. Magn., vol. MAG-23, 1987.

[29] D. Rogovin and D. J. Scalapino, "Fluctuation phenomena in tunnel junctions,"' Annals Phys., vol. 86, pp. 1-90, July 1974.

[30] R. C. Dynes, V. Narayanamurti, and J. P. Garno, "Direct measurement of quasiparicle lifetime broadening in a strong-coupled superconductor," Phys. Rev. Lett., vol. 41, p. 1509-1511, Nov. 20, 1978.

[31] I. Giaever, "Energy gap in superconductors measured by electron tunneling,"' Phys. Rev. Lett., vol, 5, pp. 147-148, Aug. 15, 1960.

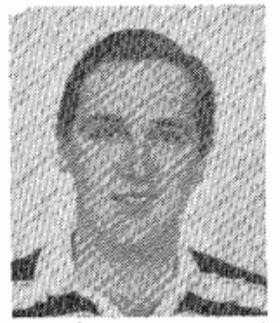

Michael J. Wengler (S'87) was born on Long Island, NY, in 1957. He received the B.A. degree with honors in physics from Swarthmore College, Swarthmore, PA, in 1978.

From 1978 to 1980 , he worked at Bell Laboratories on cooled Schottky diode receivers for millimeter radioastronomy. In 1980, he became a graduate student at the California Institute of Technology, Pasadena. Since then, he has worked on SIS receivers for millimeter and submillimeter radioastronomy. Receivers he has worked on are used at the Owens Valley Radio Observatory, and will soon be used at the Caltech Submillimeter Observatory on Mauna Kea, HI. His work has included SIS fabrication (with Bell Labs), the construction of an SIS mixer with integrated bowtie antenna, the creation of a library of computer programs for numerical computations from the quantum mixer theory, and work on advancing the quantum mixer theory. Currently, he is working towards the Ph.D. degree in applied physics at Caltech.

Mr. Wengler is a member of the Society of Photo-Optical Instrumentation Engineers and a student member of the American Physical Society.

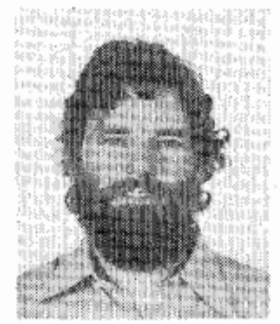

David P. Woody (M'84) was born in Santa Monica, CA, on October 30, 1946. He received the B.A. and Ph.D. degrees in physics from the University of California, Berkeley, in 1968 and 1975, respectively.

He worked on balloon-based measurements of the spectrum of the Cosmic Microwave Background from 1971 until 1977. In 1978 he joined the Math, Physics, and Astronomy Department of the California Institute of Technology, Pasadena, where he worked on the development of superconductor-insulator-superconductor tunnel junction millimeter wave receivers in collaboration with Bell Laboratories. He is currently a member of the professional staff at the California Institute of Technology's Owens Valley Radio Observatory, and is involved in many aspects of the development and operation of the Owens Valley Millimeter Interferometer. 